\title{
THE SHARP WEIGHTED BOUND FOR MULTILINEAR MAXIMAL FUNCTIONS AND CALDERÓN-ZYGMUND OPERATORS
}

\author{
KANGWEI LI, KABE MOEN, AND WENCHANG SUN
}

\begin{abstract}
We investigate the weighted bounds for multilinear maximal functions and Calderón-Zygmund operators from $L^{p_{1}}\left(w_{1}\right) \times \cdots \times$ $L^{p_{m}}\left(w_{m}\right)$ to $L^{p}\left(v_{\vec{w}}\right)$, where $1<p_{1}, \cdots, p_{m}<\infty$ with $1 / p_{1}+\cdots+1 / p_{m}=$ $1 / p$ and $\vec{w}$ is a multiple $A_{\vec{P}}$ weight. We prove the sharp bound for the multilinear maximal function for all such $p_{1}, \ldots, p_{m}$ and prove the sharp bound for $m$-linear Calderón-Zymund operators when $p \geq 1$.
\end{abstract}

\section{Introduction and Main Results}

A weight is a non-negative locally integrable function. Given $p, 1<p<$ $\infty$, an $A_{p}$ weight is one that satisfies the following

$$
[w]_{A_{p}}=\sup _{Q}\left(\frac{1}{|Q|} \int_{Q} w\right)\left(\frac{1}{|Q|} \int_{Q} w^{1-p^{\prime}}\right)^{p-1}<\infty .
$$

It is well known that the Hardy-Littlewood maximal operator and CalderónZygmund operators are bounded on $L^{p}(w)$ when $w \in A_{p}$. The sharp dependence for the Hardy-Littlewood maximal function is given by

$$
\|M\|_{L^{p}(w) \rightarrow L^{p}(w)} \leq C_{n, p}[w]_{A_{p}}^{\frac{p^{\prime}}{p}} .
$$

Inequality (1.1) was first proven by Buckley [1]. (We refer the reader to [11] for a beautiful proof of this inequality and a summary of the history.) Later is was proven by Hytönen [8] that if $T$ is a Calderón-Zygmund operator then

$$
\|T\|_{L^{p}(w) \rightarrow L^{p}(w)} \leq C_{n, p, T}[w]_{A_{p}}^{\max \left(1, \frac{p^{\prime}}{p}\right)} .
$$

Again, we refer the reader to [9, 12] for the background material and further references. In this article we prove the the multilinear analogs of inequalities (1.1) and (1.2). We begin with a few definitions.

First, let us define multiple $A_{\vec{P}}$ weights. In [13], Lerner, Ombrosi, Pérez, Torres and Trujillo-González introduced the theory of multiple $A_{\vec{P}}$ weights.

2010 Mathematics Subject Classification. 42B20, 42B25, 47H60.

Key words and phrases. multiple weights; multilinear maximal function; multilinear Calderón-Zygmund operators; weighted estimates.

The second author is partially supported by the NSF under grant 1201504. The third author is supported partially by the National Natural Science Foundation of China (10990012) and the Research Fund for the Doctoral Program of Higher Education. 
Definition 1.1. Let $\vec{P}=\left(p_{1}, \cdots, p_{m}\right)$ with $1 \leq p_{1}, \cdots, p_{m}<\infty$ and $1 / p_{1}+$ $\cdots+1 / p_{m}=1 / p$. Given $\vec{w}=\left(w_{1}, \cdots, w_{m}\right)$, set

$$
v_{\vec{w}}=\prod_{i=1}^{m} w_{i}^{p / p_{i}}
$$

We say that $\vec{w}$ satisfies the multilinear $A_{\vec{P}}$ condition if

$$
[\vec{w}]_{A_{\vec{P}}}:=\sup _{Q}\left(\frac{1}{|Q|} \int_{Q} v_{\vec{w}}\right) \prod_{i=1}^{m}\left(\frac{1}{|Q|} \int_{Q} w_{i}^{1-p_{i}^{\prime}}\right)^{p / p_{i}^{\prime}}<\infty,
$$

where $[\vec{w}]_{A_{\vec{P}}}$ is called the $A_{\vec{P}}$ constant of $\vec{w}$. When $p_{i}=1,\left(\frac{1}{|Q|} \int_{Q} w_{i}^{1-p_{i}^{\prime}}\right)^{1 / p_{i}^{\prime}}$ is understood as $\left(\inf _{Q} w_{i}\right)^{-1}$.

It is easy to see that in the linear case (that is, if $m=1$ ) $[\vec{w}]_{A_{\vec{P}}}=[w]_{A_{p}}$ is the usual $A_{p}$ constant. In [13], it was shown that for $1<p_{1}, \cdots, p_{m}<\infty$, $\vec{w} \in A_{\vec{P}}$ if and only if $w_{i}^{1-p_{i}^{\prime}} \in A_{m p_{i}^{\prime}}$ and $v_{\vec{w}} \in A_{m p}$.

Given $\vec{f}=\left(f_{1}, \cdots, f_{m}\right)$, we define the multilinear maximal function by

$$
\mathcal{M}(\vec{f})=\sup _{Q \ni x} \prod_{i=1}^{m} \frac{1}{|Q|} \int_{Q}\left|f_{i}\right| .
$$

In [13], the authors proved that $\vec{w} \in A_{\vec{P}}$ if and only if

$$
\|\mathcal{M}(\vec{f})\|_{L^{p}\left(v_{\vec{w}}\right)} \leq C \prod_{i=1}^{m}\left\|f_{i}\right\|_{L^{p_{i}\left(w_{i}\right)}} .
$$

Recall that inequality (1.1) is sharp in the sense that the exponent on $[w]_{A_{p}}$ cannot be improved. The analogous question for the operator $\mathcal{M}$ has remained open. In [5], by mixed estimates involving $A_{\infty}$, Damián, Lerner and Pérez proved the following result.

Theorem A. [5, Theorem 1.2] Let $1<p_{i}<\infty, i=1, \cdots, m$ and $1 / p=$ $1 / p_{1}+\cdots+1 / p_{m}$. Denote by $\alpha=\alpha\left(p_{1}, \cdots, p_{m}\right)$ the best possible power in

$$
\|\mathcal{M}(\vec{f})\|_{L^{p}\left(v_{\vec{w}}\right)} \leq C_{m, n, \vec{P}}[\vec{w}]_{A_{\vec{P}}}^{\alpha} \prod_{i=1}^{m}\left\|f_{i}\right\|_{L^{p_{i}\left(w_{i}\right)}} .
$$

Then the following results hold:

(1) for all $1<p_{1}, \cdots, p_{m}<\infty, \frac{m}{m p-1} \leq \alpha \leq \frac{1}{p}\left(1+\sum_{i=1}^{m} \frac{1}{p_{i}-1}\right)$.

(2) if $p_{1}=p_{2}=\cdots=p_{m}=r>1$, then $\alpha=\frac{m}{r-1}$.

Interestingly, the mixed estimates involving $A_{\infty}$ do not yield the sharp dependence on the constant $[\vec{w}]_{A_{\vec{P}}}$. The sharp bound along the diagonal is obtained using similar methods to those found in [11] and these techniques only seem to work along the diagonal. In this paper we find the optimal power on $[\vec{w}]_{A_{\vec{P}}}$ for the full range of exponents, $1<p_{1}, \ldots, p_{m}<\infty$. 


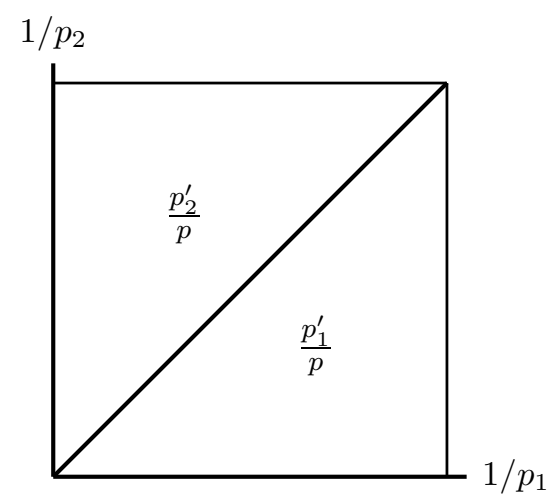

FiguRE 1. The sharp exponents on $[\vec{w}]_{A_{\vec{P}}}$ for the bilinear maximal function.

Theorem 1.2. Suppose $1<p_{1}, \ldots, p_{m}<\infty, 1 / p=1 / p_{1}+\cdots+1 / p_{m}$, and $\vec{w} \in A_{\vec{P}}$. Then

$$
\|\mathcal{M}(\vec{f})\|_{L^{p}\left(v_{\vec{w}}\right)} \leq C_{m, n, \vec{P}}[\vec{w}]_{A_{\vec{P}}}^{\max \left(\frac{p_{1}^{\prime}}{p}, \cdots, \frac{p_{m}^{\prime}}{p}\right)} \prod_{i=1}^{m}\left\|f_{i}\right\|_{L^{p_{i}\left(w_{i}\right)}} .
$$

Moreover the exponent $[\vec{w}]_{A_{\vec{P}}}$ is the best possible.

We emphasize that our bounds (1.4) not only improves those in Theorem A, but is also the best possible. See Figure 1 for a visualization of the bilinear case.

Next we turn to study weighted bounds of multilinear Calderón-Zygmund operators. The theory of multilinear Calderón-Zygmund operators originated in the works of Coifman and Meyer [3, 4] and was later developed by Christ and Journé [2], Kenig and Stein [10], and Grafakos and Torres [7. The last work provides a comprehensive account of general multilinear Calderón-Zygmund operators which we follow in this paper.

Definition 1.3. Let $T$ be a multilinear operator initially defined on the $m$ fold product of Schwartz spaces and taking values into the space of tempered distributions,

$$
T: \mathscr{S}\left(\mathbb{R}^{n}\right) \times \cdots \times \mathscr{S}\left(\mathbb{R}^{n}\right) \rightarrow \mathscr{S}^{\prime}\left(\mathbb{R}^{n}\right) .
$$

we say that $T$ is an m-linear Calderón-Zygmund operator if, for some $1 \leq$ $q_{i}<\infty$, it extends to a bounded multilinear operator from $L^{q_{1}} \times \cdots \times L^{q_{m}}$ to $L^{q}$, where $1 / q_{1}+\cdots+1 / q_{m}=1 / q$, and if there exists a function $K$, defined off the diagonal $x=y_{1}=\cdots=y_{m}$ in $\left(\mathbb{R}^{n}\right)^{m+1}$, satisfying

$$
T\left(f_{1}, \cdots, f_{m}\right)=\int_{\left(\mathbb{R}^{n}\right)^{m}} K\left(x, y_{1}, \cdots, y_{m}\right) f_{1}\left(y_{1}\right) \cdots f_{m}\left(y_{m}\right) d y_{1} \cdots d y_{m}
$$


for all $x \notin \bigcap_{j=1}^{m} \operatorname{supp} f_{i}$;

$$
\left|K\left(y_{0}, y_{1}, \cdots, y_{m}\right)\right| \leq \frac{A}{\left(\sum_{k, l=0}^{m}\left|y_{k}-y_{l}\right|\right)^{m n}}
$$

and

$$
\left|K\left(y_{0}, \cdots, y_{i}, \cdots, y_{m}\right)-K\left(y_{0}, \cdots, y_{i}^{\prime}, \cdots, y_{m}\right)\right| \leq \frac{A\left|y_{i}-y_{i}^{\prime}\right|^{\varepsilon}}{\left(\sum_{k, l=0}^{m}\left|y_{k}-y_{l}\right|\right)^{m n+\varepsilon}}
$$

for some $A, \varepsilon>0$ and all $0 \leq i \leq m$, whenever $\left|y_{i}-y_{i}^{\prime}\right| \leq \frac{1}{2} \max _{0 \leq k \leq m} \mid y_{i}-$ $y_{k} \mid$.

It was shown in [7] that if $1 / r_{1}+\cdots+1 / r_{m}=1 / r$, then an $m$-linear Calderón-Zygmund operator $T$ is bounded from $L^{r_{1}} \times \cdots \times L^{r_{m}}$ to $L^{r}$ when $1<r_{i}<\infty$ for all $i=1, \cdots, m$; and $T$ is bounded from $L^{r_{1}} \times \cdots \times L^{r_{m}}$ to $L^{r, \infty}$ when at least one $r_{i}=1$. In particular, $T$ is bounded from $L^{1} \times \cdots \times L^{1}$ to $L^{1 / m, \infty}$.

A weighted theory for $m$-linear Calderón-Zygmund operators was developed in [13], where it was shown that such operators are bounded from $L^{p_{1}}\left(w_{1}\right) \times \cdots \times L^{p_{m}}\left(w_{m}\right)$ to $L^{p}\left(v_{\vec{w}}\right)$ when $\vec{w} \in A_{\vec{P}}$. In [5], the authors proved a multilinear version of the $A_{2}$ conjecture. Specifically, for $p_{1}=$ $\cdots=p_{m}=m+1$, it was shown that

$$
\|T(\vec{f})\|_{L^{p}\left(v_{\vec{w}}\right)} \lesssim[\vec{w}]_{A_{\vec{P}}} \prod_{i=1}^{m}\left\|f_{i}\right\|_{L^{p_{i}\left(w_{i}\right)}},
$$

where the estimate for the power of $[\vec{w}]_{\vec{P}}$ is the best possible.

Due to the lack of an appropriate extrapolation theorem for multilinear operators with multiple weights, let alone a version with good constants, the sharp estimate is unknown for any other choices of $p_{i}$. In this paper, we give a sharp estimate for the case of $p \geq 1$. Specifically, we prove the following Theorem, again we refer the reader to Figure 2 for a visualization of the bilinear case.

Theorem 1.4. Let $T$ be a multilnear Calderón-Zygmund operator, $\vec{P}=$ $\left(p_{1}, \cdots, p_{m}\right)$ with $1<p_{1}, \cdots, p_{m}<\infty$ and $1 / p_{1}+\cdots+1 / p_{m}=1 / p \leq 1$. If $\vec{w}=\left(w_{1}, \cdots, w_{m}\right) \in A_{\vec{P}}$, then

$$
\|T(\vec{f})\|_{L^{p}\left(v_{\vec{w}}\right)} \leq C_{n, m, \vec{P}, T}[\vec{w}]_{A_{\vec{P}}}^{\max \left(1, \frac{p_{1}^{\prime}}{p}, \ldots, \frac{p_{m}^{\prime}}{p}\right)} \prod_{i=1}^{m}\left\|f_{i}\right\|_{L^{p_{i}}\left(w_{i}\right)} .
$$

Moreover, for certain multilinear operators the exponent on $[\vec{w}]_{A_{\vec{P}}}$ is the best possible

In order to prove Theorem 1.4 we will approximate multilinear CalderónZygmund operators by positive dyadic operators. The result of Damián, Lerner and Pérez [5] states the following (see Section 2 for pertinent definition). 


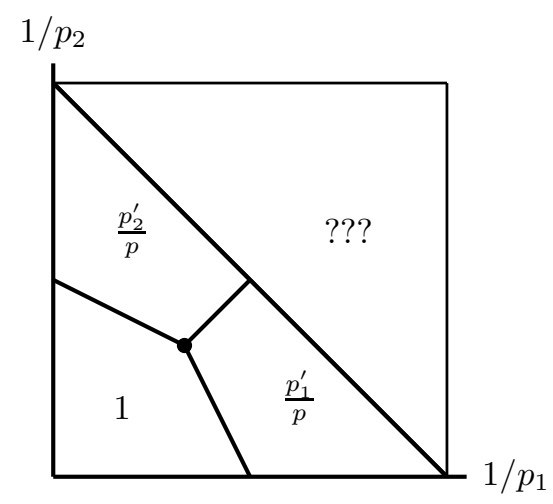

FiguRE 2. The sharp exponents on $[\vec{w}]_{A_{\vec{P}}}$ for bilinear Calderón-Zygmund operators when $p \geq 1$

Theorem 1.5. [5, Theorem 1.4] Let $T$ be a multilinear Calderón-Zygmund operator and let $\mathcal{X}$ be a Banach function space over $\mathbb{R}^{n}$ equipped with Lebesgue measure. Then, for any appropriate $\vec{f}$,

$$
\|T(\vec{f})\|_{\mathcal{X}} \leq C_{T, m, n} \sup _{\mathscr{D}, \mathcal{S}}\left\|A_{\mathscr{D}, \mathcal{S}}(|\vec{f}|)\right\|_{\mathcal{X}} .
$$

When $p \geq 1, \mathcal{X}=L^{p}(v)$ is a Banach space. However, for $0<p<1$ it is not. Since the $m$-linear operators map into $L^{p}$ for $p>1 / m$ we are are unable to obtain the full range. It is an interesting question as to whether the same decomposition can be obtained for non Banach spaces such as $L^{p}$ for $0<p<1$. Moreover, we believe that inequality (1.5) should hold for all $1<p_{1}, \ldots, p_{m}<\infty$.

We will actually prove the estimate in Theorem 1.4 for the sparse operators $A_{\mathscr{D}, \mathcal{S}}$. For these operators, the main techniques are an extension of those found in 15, in which the second author proved the sharp weighted bound for linear Calderón-Zygmund operators without extrapolation.

The rest of this article is devoted to the following. In Section 2 we state some brief preliminary material. In Section 3 we will prove the main estimates in Theorems 1.2 and 1.4 and in Section 4 we will provide examples to show that our results are sharp.

\section{Preliminaries}

Recall that the standard dyadic grid in $\mathbb{R}^{n}$ consists of the cubes

$$
2^{-k}\left([0,1)^{n}+j\right), \quad k \in \mathbb{Z}, j \in \mathbb{Z}^{n} .
$$

Denote the standard grid by $\mathcal{D}$.

By a general dyadic grid $\mathscr{D}$ we mean a collection of cubes with the following properties: (i) for any $Q \in \mathscr{D}$ its sidelength, $l_{Q}$, is of the form $2^{k}, k \in \mathbb{Z}$; (ii) $Q \cap R \in\{Q, R, \emptyset\}$ for any $Q, R \in \mathscr{D}$; (iii) the cubes of a fixed sidelength $2^{k}$ form a partition of $\mathbb{R}^{n}$. 
Now we define the dyadic maximal function with respect to arbitrary weight

$$
M_{w}^{\mathscr{D}} f(x)=\sup _{Q \ni x, Q \in \mathscr{D}} \frac{1}{w(Q)} \int_{Q}|f| w .
$$

It is well-known that

$$
\left\|M_{w}^{\mathscr{D}} f\right\|_{L^{p}(w)} \leq p^{\prime}\|f\|_{L^{p}(w)}, \quad 1<p<\infty
$$

we refer the readers to [15] for a proof.

We will also need the notion of a sparse family of cubes. Given a dyadic grid $\mathscr{D}$ we say that a family $\mathcal{S}$ is sparse if there are disjoint majorizing subsets, that is, for each $Q \in \mathcal{S}$ there exists $E_{Q} \subset Q$ such that $\left\{E_{Q}\right\}_{Q \in \mathcal{S}}$ is pairwise disjoint and $\left|E_{Q}\right| \geq \frac{1}{2}|Q|$. Sparse families have long played a role in Calderón-Zygmund theory, our definition can be found in [9]. Finally, in [5], the multilinear sparse operators,

$$
A_{\mathscr{D}, \mathcal{S}}(\vec{f})=\sum_{Q \in \mathcal{S}}\left(\prod_{i=1}^{m} \frac{1}{|Q|} \int_{Q} f_{i}\right) \chi_{Q}
$$

were defined and used to approximate multlinear Calderón-Zygmund operators (see Theorem 1.5).

\section{Proof of Theorems 1.2 And 1.4}

First, we give a proof for Theorem 1.2 .

Proof of Theorem 1.2. In [5], the authors proved that there exists $2^{n}$ families of dyadic grids $\mathscr{D}_{\beta}$ such that

$$
\mathcal{M}(\vec{f})(x) \leq 6^{m n} \sum_{\beta=1}^{2^{n}} \mathcal{M}^{\mathscr{D}_{\beta}}(\vec{f})(x),
$$

where

$$
\mathcal{M}^{\mathscr{D}_{\beta}}(\vec{f})(x)=\sup _{Q \ni x, Q \in \mathscr{D}_{\beta}} \prod_{i=1}^{m} \frac{1}{|Q|} \int_{Q}\left|f_{i}\right| .
$$

Without loss of generality, it suffices to prove that

$$
\left\|\mathcal{M}^{\mathscr{D}}(\overrightarrow{f \sigma})\right\|_{L^{p}\left(v_{\vec{w}}\right)} \leq C_{m, n, \vec{P}}[\vec{w}]_{A_{\vec{P}}}^{\max _{i}\left(\frac{p_{i}}{p}\right)} \prod_{i=1}^{m}\left\|f_{i}\right\|_{L^{p_{i}}\left(\sigma_{i}\right)} .
$$

for a general dyadic grid $\mathscr{D}$, and $\mathcal{M}^{\mathscr{D}}(\overrightarrow{f \sigma})=\mathcal{M}^{\mathscr{D}}\left(f_{1} \sigma_{1}, \ldots, f_{m} \sigma_{m}\right)$. Moreover, it was shown in [5, Lemma 2.2] that there exists a sparse subset $\mathcal{S} \subset \mathscr{D}$ such that

$$
\mathcal{M}^{\mathscr{D}}(\overrightarrow{f \sigma}) \lesssim \sum_{Q \in \mathcal{S}} \prod_{i=1}^{m}\left(\frac{1}{|Q|} \int_{Q}\left|f_{i}\right| \sigma_{i}\right) \chi_{E_{Q}}
$$


Without loss of generality, assume that $p_{1}=\min \left\{p_{1}, \cdots, p_{m}\right\}$. We have

$$
\begin{aligned}
\int_{\mathbb{R}^{n}} \mathcal{M}^{\mathscr{D}}(\vec{f} \sigma)^{p} v_{\vec{w}} \lesssim \sum_{Q \in \mathcal{S}} \prod_{i=1}^{m}\left(\frac{1}{|Q|} \int_{Q}\left|f_{i}\right| \sigma_{i}\right)^{p} v_{\vec{w}}(Q) \\
=\sum_{Q \in \mathcal{S}} \frac{v_{\vec{w}}(Q)^{p_{1}^{\prime}} \prod_{i=1}^{m} \sigma_{i}(Q)^{p p_{1}^{\prime} / p_{i}^{\prime}}}{|Q|^{m p p_{1}^{\prime}}}\left(\prod_{i=1}^{m} \int_{Q}\left|f_{i}\right| \sigma_{i}\right)^{p} \\
\cdot \frac{|Q|^{m p\left(p_{1}^{\prime}-1\right)}}{v_{\vec{w}}(Q)^{p_{1}^{\prime}-1} \prod_{i=1}^{m} \sigma_{i}(Q)^{p p_{1}^{\prime} / p_{i}^{\prime}}} \\
\leq[\vec{w}]_{A_{\vec{P}}}^{p_{1}^{\prime}} \sum_{Q \in \mathcal{S}} \frac{2^{m p\left(p_{1}^{\prime}-1\right)}\left|E_{Q}\right|^{m p\left(p_{1}^{\prime}-1\right)}}{v_{\vec{w}}(Q)^{p_{1}^{\prime}-1} \prod_{i=1}^{m} \sigma_{i}(Q)^{p p_{1}^{\prime} / p_{i}^{\prime}}} \cdot\left(\prod_{i=1}^{m} \int_{Q}\left|f_{i}\right| \sigma_{i}\right)^{p} .
\end{aligned}
$$

By Hölder's inequality, we have

$$
\begin{aligned}
\left|E_{Q}\right| & =\int_{E_{Q}} v_{\vec{w}}^{\frac{1}{m p}} \sigma_{1}^{\frac{1}{m p_{1}^{\prime}}} \cdots \sigma_{m}^{\frac{1}{m p_{m}^{\prime}}} \\
& \leq v_{\vec{w}}\left(E_{Q}\right)^{\frac{1}{m p}} \sigma_{1}\left(E_{Q}\right)^{\frac{1}{m p_{1}^{\prime}}} \cdots \sigma_{m}\left(E_{Q}\right)^{\frac{1}{m p_{m}^{\prime}}} .
\end{aligned}
$$

Therefore,

$$
\left|E_{Q}\right|^{m p\left(p_{1}^{\prime}-1\right)} \leq v_{\vec{w}}\left(E_{Q}\right)^{p_{1}^{\prime}-1} \sigma_{1}\left(E_{Q}\right)^{\frac{p\left(p_{1}^{\prime}-1\right)}{p_{1}^{\prime}}} \cdots \sigma_{m}\left(E_{Q}\right)^{\frac{p\left(p_{1}^{\prime}-1\right)}{p_{m}^{\prime}}}
$$

and

$$
\frac{p\left(p_{1}^{\prime}-1\right)}{p_{i}^{\prime}}-\frac{p}{p_{i}}=\frac{p p_{1}^{\prime}}{p_{i}^{\prime}}-p \geq 0 .
$$

Since $E_{Q} \subset Q$, we have

$$
v_{\vec{w}}\left(E_{Q}\right)^{p_{1}^{\prime}-1} \leq v_{\vec{w}}(Q)^{p_{1}^{\prime}-1}
$$

and hence

$$
\sigma_{i}\left(E_{Q}\right)^{\frac{p\left(p_{1}^{\prime}-1\right)}{p_{i}^{\prime}}-\frac{p}{p_{i}}} \leq \sigma_{i}(Q)^{\frac{p p_{1}^{\prime}}{p_{i}^{\prime}}-p}, \quad i=1, \cdots, m .
$$

It follows that

$$
\begin{aligned}
& \sum_{Q \in \mathcal{S}} \frac{\left|E_{Q}\right|^{m p\left(p_{1}^{\prime}-1\right)}}{v_{\vec{w}}(Q)^{p_{1}^{\prime}-1} \prod_{i=1}^{m} \sigma_{i}(Q)^{p p_{1}^{\prime} / p_{i}^{\prime}}} \cdot\left(\prod_{i=1}^{m} \int_{Q}\left|f_{i}\right| \sigma_{i}\right)^{p} \\
& \leq \sum_{Q \in \mathcal{S}} \prod_{i=1}^{m}\left(\frac{1}{\sigma_{i}(Q)} \int_{Q}\left|f_{i}\right| \sigma_{i}\right)^{p} \sigma_{i}\left(E_{Q}\right)^{p / p_{i}} \\
& \leq \prod_{i=1}^{m}\left(\sum_{Q \in \mathcal{S}}\left(\frac{1}{\sigma_{i}(Q)} \int_{Q}\left|f_{i}\right| \sigma_{i}\right)^{p_{i}} \sigma_{i}\left(E_{Q}\right)\right)^{p / p_{i}} \\
& \quad \leq \prod_{i=1}^{m}\left\|M_{\sigma_{i}}^{\mathscr{D}}\left(f_{i}\right)\right\|_{L^{p_{i}}\left(\sigma_{i}\right)}^{p}
\end{aligned}
$$




$$
\lesssim \prod_{i=1}^{m}\left\|f_{i}\right\|_{L^{p_{i}\left(\sigma_{i}\right)}}^{p}
$$

Hence

$$
\left\|\mathcal{M}^{\mathscr{D}}(\vec{f})\right\|_{L^{p}\left(v_{\vec{w}}\right)} \leq C_{m, n, \vec{P}}[\vec{w}]_{A_{\vec{P}}}^{\max _{i}\left(\frac{p_{i}^{\prime}}{p}\right)} \prod_{i=1}^{m}\left\|f_{i}\right\|_{L^{p_{i}\left(w_{i}\right)}} .
$$

This completes the proof.

We now turn our attention to the proof of Theorem 1.4. First, we note the following symmetry of $A_{\vec{P}}$ weights.

Lemma 3.1. Suppose that $\vec{w}=\left(w_{1}, \cdots, w_{m}\right) \in A_{\vec{P}}$ and that $1<p, p_{1}, \cdots$, $p_{m}<\infty$ with $1 / p_{1}+\cdots+1 / p_{m}=1 / p$. Then $\vec{w}^{i}:=\left(w_{1}, \cdots, w_{i-1}, v_{\vec{w}}^{1-p^{\prime}}\right.$, $\left.w_{i+1}, \cdots, w_{m}\right) \in A_{\vec{P}^{i}}$ with $\vec{P}^{i}=\left(p_{1}, \cdots, p_{i-1}, p^{\prime}, p_{i+1}, \cdots, p_{m}\right)$ and

$$
\left[\vec{w}^{i}\right]_{A_{\vec{P} i}}=[\vec{w}]_{A_{\vec{P}}}^{p_{i}^{\prime} / p} .
$$

Proof. We will prove the conclusion for $i=1$; the other cases are similar. Notice that

$$
1 / p^{\prime}+1 / p_{2}+\cdots+1 / p_{m}=1 / p_{1}^{\prime}
$$

and

$$
v_{\vec{w}}^{\left(1-p^{\prime}\right) p_{1}^{\prime} / p^{\prime}} \cdot w_{2}^{p_{1}^{\prime} / p_{2}} \cdots w_{m}^{p_{1}^{\prime} / p_{m}}=w_{1}^{1-p_{1}^{\prime}}
$$

By the definition of multiple $A_{\vec{P}}$ constant, we have

$$
\begin{aligned}
{\left[\vec{w}^{1}\right]_{A_{\vec{P} 1}}=\sup _{Q}\left(\frac{1}{|Q|} \int_{Q} w_{1}^{1-p_{1}^{\prime}}\right) \cdot\left(\frac{1}{|Q|} \int_{Q}\left(v_{\vec{w}}^{1-p^{\prime}}\right)^{1-p}\right)^{p_{1}^{\prime} / p} } \\
\quad \times \prod_{i=2}^{m}\left(\frac{1}{|Q|} \int_{Q} w_{i}^{1-p_{i}^{\prime}}\right)^{p_{1}^{\prime} / p_{i}^{\prime}} \\
=[\vec{w}]_{A_{\vec{P}}}^{p_{1}^{\prime} / p}
\end{aligned}
$$

By Theorem 1.5 we reduce our problem to consider the behavior of the operator $A_{\mathscr{D}, \mathcal{S}}$. For these operators we have the following Theorem, which holds for all $1<p_{1}, \ldots, p_{m}<\infty$.

Theorem 3.2. Suppose that $1<p_{1}, \cdots, p_{m}<\infty$ with $1 / p_{1}+\cdots+1 / p_{m}=$ $1 / p$ and $\vec{w} \in A_{\vec{P}}$. Then

$$
\left\|A_{\mathscr{D}, \mathcal{S}}(\vec{f})\right\|_{L^{p}\left(v_{\vec{w}}\right)} \lesssim[\vec{w}]_{A_{\vec{P}}}^{\max \left(1, \frac{p_{1}^{\prime}}{p}, \ldots, \frac{p_{m}^{\prime}}{p}\right)} \prod_{i=1}^{m}\left\|f_{i}\right\|_{L^{p_{i}}\left(w_{i}\right)} .
$$

Proof. We first consider the case when $\frac{1}{m}<p \leq 1$. In this case

$$
\int_{\mathbb{R}^{n}} A_{\mathscr{D}, \mathcal{S}}(\vec{f})^{p} v_{\vec{w}} \leq \sum_{Q \in \mathcal{S}}\left(\prod_{i=1}^{m} \frac{1}{|Q|} \int_{Q} f_{i}\right)^{p} v_{\vec{w}}(Q),
$$


which can be handled in exactly the same manner as the estimates in proof of Theorem 1.2.

Now consider the case $p \geq \max _{i} p_{i}^{\prime}$. It is sufficient to prove that

$$
\left\|A_{\mathscr{D}, \mathcal{S}}(\overrightarrow{f \sigma})\right\|_{L^{p}\left(v_{\vec{w}}\right)} \lesssim[\vec{w}]_{\vec{P}} \prod_{i=1}^{m}\left\|f_{i}\right\|_{L^{p_{i}}\left(\sigma_{i}\right)},
$$

where $\sigma_{i}=w_{i}^{1-p_{i}^{\prime}}, A_{\mathscr{D}, \mathcal{S}}(\overrightarrow{f \sigma})=A_{\mathscr{D}, \mathcal{S}}\left(f_{1} \sigma_{1}, \cdots, f_{m} \sigma_{m}\right)$, and $f_{i} \geq 0$. By duality, it suffices to estimate the $(m+1)$-linear form

$$
\int_{\mathbb{R}^{n}} A_{\mathscr{D}, \mathcal{S}}(\overrightarrow{f \sigma}) g v_{\vec{w}}=\sum_{Q \in \mathcal{S}} \int_{Q} g v_{\vec{w}} \cdot \prod_{i=1}^{m} \frac{1}{|Q|} \int_{Q} f_{i} \sigma_{i}
$$

for $g \geq 0$ belonging to $L^{p^{\prime}}\left(v_{\vec{w}}\right)$. We have

$$
\begin{aligned}
\sum_{Q \in \mathcal{S}} \int_{Q} g v_{\vec{w}} \cdot \prod_{i=1}^{m} \frac{1}{|Q|} \int_{Q} f_{i} \sigma_{i} \\
=\sum_{Q \in \mathcal{S}} \frac{v_{\vec{w}}(Q) \prod_{i=1}^{m} \sigma_{i}(Q)^{p / p_{i}^{\prime}}}{|Q|^{m p}} \cdot \frac{|Q|^{m(p-1)}}{v_{\vec{w}}(Q) \prod_{i=1}^{m} \sigma_{i}(Q)^{p / p_{i}^{\prime}}} \\
\quad \int_{Q} g v_{\vec{w}} \cdot \prod_{i=1}^{m} \int_{Q} f_{i} \sigma_{i} \\
\leq[\vec{w}]_{A_{\vec{P}}} \sum_{Q \in \mathcal{S}} \frac{|Q|^{m(p-1)}}{v_{\vec{w}}(Q) \prod_{i=1}^{m} \sigma_{i}(Q)^{p / p_{i}^{\prime}}} \cdot \int_{Q} g v_{\vec{w}} \cdot \prod_{i=1}^{m} \int_{Q} f_{i} \sigma_{i} \\
\leq[\vec{w}]_{A_{\vec{P}}} \sum_{Q \in \mathcal{S}} \frac{2^{m(p-1)}\left|E_{Q}\right|^{m(p-1)}}{v_{\vec{w}}(Q) \prod_{i=1}^{m} \sigma_{i}(Q)^{p / p_{i}^{\prime}}} \cdot \int_{Q} g v_{\vec{w}} \cdot \prod_{i=1}^{m} \int_{Q} f_{i} \sigma_{i} .
\end{aligned}
$$

By (3.1),

$$
\left|E_{Q}\right| \leq v_{\vec{w}}\left(E_{Q}\right)^{\frac{1}{m p}} \sigma_{1}\left(E_{Q}\right)^{\frac{1}{m p_{1}^{\prime}}} \cdots \sigma_{m}\left(E_{Q}\right)^{\frac{1}{m p_{m}^{\prime}}} .
$$

Since $p \geq \max _{i}\left\{p_{i}^{\prime}\right\}$ and $E_{Q} \subset Q$, we have $\sigma_{i}(Q)^{1-\frac{p}{p_{i}^{\prime}}} \leq \sigma_{i}\left(E_{Q}\right)^{1-\frac{p}{p_{i}^{\prime}}}$ for any $i=1, \cdots, m$. Therefore,

$$
\begin{aligned}
& \sum_{Q \in \mathcal{S}} \int_{Q} g v_{\vec{w}} \cdot \prod_{i=1}^{m} \frac{1}{|Q|} \int_{Q} f_{i} \sigma_{i} \\
\leq & 2^{m(p-1)}[\vec{w}]_{A_{\vec{P}}} \sum_{j, k} v_{\vec{w}}\left(E_{Q}\right)^{\frac{1}{p^{\prime}}} \prod_{i=1}^{m} \sigma_{i}\left(E_{Q}\right)^{\frac{p-1}{p_{i}^{\prime}}} \sigma_{i}(Q)^{1-\frac{p}{p_{i}^{\prime}}} \\
& \frac{1}{v_{\vec{w}}(Q)} \int_{Q} g v_{\vec{w}} \cdot \prod_{i=1}^{m} \frac{1}{\sigma_{i}(Q)} \int_{Q} f_{i} \sigma_{i} \\
\leq & 2^{m(p-1)}[\vec{w}]_{A_{\vec{P}}} \sum_{Q \in \mathcal{S}} v_{\vec{w}}\left(E_{Q}\right)^{\frac{1}{p^{\prime}}} \prod_{i=1}^{m} \sigma_{i}\left(E_{Q}\right)^{\frac{1}{p_{i}}} \frac{1}{v_{\vec{w}}(Q)} \int_{Q} g v_{\vec{w}}
\end{aligned}
$$




$$
\begin{aligned}
& \prod_{i=1}^{m} \frac{1}{\sigma_{i}(Q)} \int_{Q} f_{i} \sigma_{i} \\
\leq & 2^{m(p-1)}[\vec{w}]_{A_{\vec{P}}}\left(\sum_{Q \in \mathcal{S}}\left(\frac{1}{v_{\vec{w}}(Q)} \int_{Q} g v_{\vec{w}}\right)^{p^{\prime}} v_{\vec{w}}\left(E_{Q}\right)\right)^{1 / p^{\prime}} \\
\leq & \prod_{i=1}^{m}\left(\sum_{Q \in \mathcal{S}}\left(\frac{1}{\sigma_{i}(Q)} \int_{Q} f_{i} \sigma_{i}\right)^{p_{i}} \sigma_{i}\left(E_{Q}\right)\right)^{1 / p_{i}} \\
\lesssim 2^{m(p-1)}[\vec{w}]_{A_{\vec{P}}}\left\|M_{v_{\vec{w}}}^{\mathscr{D}}(g)\right\|_{L^{p^{\prime}}\left(v_{\vec{w}}\right)} \prod_{i=1}^{m}\left\|M_{A_{\vec{P}}}^{\mathscr{D}}\left(f_{i}\right)\right\|_{L^{p_{i}}\left(\sigma_{i}\right)} & \|\|_{L^{p^{\prime}}\left(v_{\vec{w}}\right)} \prod_{i=1}^{m}\left\|f_{i}\right\|_{L^{p_{i}}\left(\sigma_{i}\right)},
\end{aligned}
$$

where (2.1) is used in the last step. For the other cases we use duality. Notice that the operator $A_{\mathscr{D}, \mathcal{S}}$ is self adjoint as a multilinear operator, in the sense that for any $i, i=1, \ldots, m$, we have

$$
\int_{\mathbb{R}^{n}} A_{\mathscr{D}, \mathcal{S}}\left(f_{1}, \ldots, f_{m}\right) g=\int_{\mathbb{R}^{n}} A_{\mathscr{D}, \mathcal{S}}\left(f_{1}, \ldots, f_{i-1}, g, f_{i+1}, \ldots f_{m}\right) f_{i} .
$$

Without loss of generality suppose $p_{1}^{\prime} \geq \max \left(p, p_{2}^{\prime}, \ldots, p_{m}^{\prime}\right)$. Hence, by duality and self adjiontness we have

$$
\begin{aligned}
\left\|A_{\mathscr{D}, \mathcal{S}}\right\|_{L^{p_{1}}\left(w_{1}\right) \times \cdots \times L^{p_{m}}\left(w_{m}\right) \rightarrow L^{p}\left(v_{\vec{w}}\right)} & =\left\|A_{\mathscr{D}, \mathcal{S}}\right\|_{L^{p^{\prime}}\left(v_{\vec{w}}^{1-p^{\prime}}\right) \times \cdots \times L^{p_{m}}\left(w_{m}\right) \rightarrow L^{p_{1}^{\prime}}\left(w_{1}^{1-p_{1}^{\prime}}\right)} \\
& \lesssim\left[\vec{w}^{1}\right]_{\vec{P}^{1}}=[\vec{w}]_{A_{\vec{P}}}^{\frac{p_{1}^{\prime}}{p}}
\end{aligned}
$$

\section{EXAmples}

Finally, we end with some examples to show that our bounds are sharp. First we show that Theorem 1.2 is sharp. Consider the case $m=2$ (we leave it to the reader to modify the example for $m>2$ ) and suppose that we had a better exponent than the one in inequality 1.4, that is, suppose

$$
\|\mathcal{M}\|_{L^{p_{1}}\left(w_{1}\right) \times L^{p_{2}}\left(w_{2}\right) \rightarrow L^{p}\left(v_{\vec{w}}\right)} \lesssim[\vec{w}]_{A_{\vec{P}}}^{r \max \left(\frac{p_{1}^{\prime}}{p}, \frac{p_{2}^{\prime}}{p}\right)}
$$

for some $r<1$. Further suppose that $p_{1}^{\prime} \geq p_{2}^{\prime}$. For $0<\varepsilon<1$, let $f_{1}(x)=|x|^{\varepsilon-n} \chi_{B(0,1)}(x), f_{2}(x)=|x|^{\frac{\varepsilon-n}{p_{2}}} \chi_{B(0,1)}(x), w_{1}(x)=|x|^{(n-\varepsilon)\left(p_{1}-1\right)}$ and $w_{2}(x)=1$. Calculations show that

$$
\left\|f_{1}\right\|_{L^{p_{1}}\left(w_{1}\right)} \simeq \varepsilon^{-1 / p_{1}},\left\|f_{2}\right\|_{L^{p_{2}\left(w_{2}\right)}} \simeq \varepsilon^{-1 / p_{2}}, v_{\vec{w}}(x)=|x|^{(n-\varepsilon) \frac{p}{p_{1}^{\prime}}}
$$

and

$$
[\vec{w}]_{A_{\vec{P}}} \simeq \varepsilon^{-p / p_{1}^{\prime}}
$$


For $x \in B(0,1)$ we have

$$
\begin{aligned}
\mathcal{M}\left(f_{1}, f_{2}\right)(x) & \gtrsim \frac{1}{|x|^{n}} \int_{B(0,|x|)}\left|y_{1}\right|^{\varepsilon-n} d y_{1} \cdot \frac{1}{|x|^{n}} \int_{B(0,|x|)}\left|y_{2}\right|^{\frac{\varepsilon-n}{p_{2}}} d y_{2} \\
& \gtrsim \frac{f_{1}(x) f_{2}(x)}{\varepsilon \cdot\left(\frac{\varepsilon-n}{p_{2}}+n\right)} \gtrsim \frac{f_{1}(x) f_{2}(x)}{\varepsilon} .
\end{aligned}
$$

Hence,

$$
\begin{aligned}
\left\|\mathcal{M}\left(f_{1}, f_{2}\right)\right\|_{L^{p}\left(v_{\vec{w}}\right)} & \gtrsim \frac{1}{\varepsilon}\left(\int_{B(0,1)}|x|^{(\varepsilon-n)\left(p+\frac{p}{p_{2}}-\frac{p}{p_{1}^{\prime}}\right)} d x\right)^{1 / p} \\
& \simeq \frac{1}{\varepsilon}\left(\int_{0}^{1} x^{\varepsilon-1} d x\right)^{1 / p} \\
& =\frac{1}{\varepsilon}\left(\frac{1}{\varepsilon}\right)^{1 / p} .
\end{aligned}
$$

Combining this with inequality (4.1) we see for some $r<1$,

$$
\left(\frac{1}{\varepsilon}\right)^{1+\frac{1}{p}} \lesssim\left(\frac{1}{\varepsilon}\right)^{r+\frac{1}{p}}
$$

which is impossible as $\varepsilon \rightarrow 0$.

Next we show that Theorem 1.4 is sharp. Recall that for $i=1, \cdots, n$, the $m$-linear $i$ th Riesz transform is defined by

$$
R_{i}(\vec{f})(x)=p . v \cdot \int_{\left(\mathbb{R}^{n}\right)^{m}} \frac{\sum_{j=1}^{m}\left(x_{i}-\left(y_{j}\right)_{i}\right)}{\left(\sum_{j=1}^{m}\left|x-y_{j}\right|^{2}\right)^{(n m+1) / 2}} f_{1}\left(y_{1}\right) \cdots f_{m}\left(y_{m}\right) d y_{1} \cdots d y_{m},
$$

where $\left(y_{j}\right)_{i}$ denotes the $i$ th coordinate of $y_{j}$.

Suppose that $m=2, p_{1}^{\prime} \geq p_{2}^{\prime}$ and $p_{1}^{\prime} \geq p$. Let

$$
\begin{aligned}
U & =\left\{x \in \mathbb{R}^{n}:|x| \leq 1,0<x_{i} \leq x_{1}, i=2, \cdots, n\right\}, \\
V & =\left\{x \in \mathbb{R}^{n}:|x| \leq 1, x_{i} \leq 0, i=1, \cdots, n\right\} .
\end{aligned}
$$

For $0<\varepsilon<1$, let $f_{1}(x)=|x|^{\varepsilon-n} \chi_{V}(x), f_{2}(x)=|x|^{\frac{\varepsilon-n}{p_{2}}} \chi_{V}(x), w_{1}(x)=$ $|x|^{(n-\varepsilon)\left(p_{1}-1\right)}$ and $w_{2}(x)=1$. For $x \in U$ and $y_{j} \in V$ with $\left|y_{j}\right| \leq|x|$, we have

$$
\frac{\sum_{j=1}^{2}\left(x_{1}-\left(y_{j}\right)_{1}\right)}{\left(\sum_{j=1}^{2}\left|x-y_{j}\right|^{2}\right)^{1 / 2}} \geq \frac{2 \frac{|x|}{\sqrt{n}}}{4|x|} \gtrsim 1 \text {. }
$$

Therefore,

It follows that

$$
\frac{\sum_{j=1}^{2}\left(x_{1}-\left(y_{j}\right)_{1}\right)}{\left(\sum_{j=1}^{2}\left|x-y_{j}\right|^{2}\right)^{(2 n+1) / 2}} \gtrsim \frac{1}{|x|^{2 n}}
$$

$$
\begin{aligned}
R_{1}(\vec{f})(x) & =p \cdot v \cdot \int_{\left(\mathbb{R}^{n}\right)^{2}} \frac{\sum_{j=1}^{2}\left(x_{i}-\left(y_{j}\right)_{i}\right)}{\left(\sum_{j=1}^{2}\left|x-y_{j}\right|^{2}\right)^{(2 n+1) / 2}} f_{1}\left(y_{1}\right) f_{2}\left(y_{2}\right) d y_{1} d y_{2} \\
& \gtrsim \int_{\left|y_{1}\right| \leq|x|} \int_{\left|y_{2}\right| \leq|x|} \frac{1}{|x|^{2 n}\left|y_{1}\right|^{\varepsilon-n} \cdot\left|y_{2}\right|^{\frac{\varepsilon-n}{p_{2}}}} d y_{1} d y_{2}
\end{aligned}
$$




$$
\gtrsim \frac{1}{\varepsilon} f_{1}(x) f_{2}(x) \text {. }
$$

Hence

$$
\begin{aligned}
\left\|R_{1}(\vec{f})\right\|_{L^{p}\left(v_{\vec{w}}\right)} & \gtrsim \frac{1}{\varepsilon}\left(\int_{U}|x|^{(\varepsilon-n)\left(p+p / p_{2}-p / p_{1}^{\prime}\right)} d x\right)^{1 / p} \\
& =\frac{1}{\varepsilon}\left(\int_{U}|x|^{\varepsilon-n} d x\right)^{1 / p} \\
& \gtrsim \frac{1}{\varepsilon}\left(\int_{\{|x| \leq 1\}}|x|^{\varepsilon-n} d x\right)^{1 / p} \quad \text { (by symmetry) } \\
& \gtrsim\left(\frac{1}{\varepsilon}\right)^{1+1 / p} .
\end{aligned}
$$

Then by similar arguments as the above we can show that the exponent is sharp when $\max \left(p_{1}^{\prime}, p_{2}^{\prime}\right) \geq p \geq 1$. When $p>\max \left(p_{1}^{\prime}, p_{2}^{\prime}\right)$. Again, suppose that $p_{1}^{\prime} \geq p_{2}^{\prime}$. We consider the adjoint in the first variable, $\left(R_{1}\right)^{1, *}$. Notice that

$$
\left(R_{1}\right)^{1, *}\left(f_{1}, f_{2}\right)(x)=\int_{\left(\mathbb{R}^{n}\right)^{2}} \frac{2\left(y_{1}\right)_{1}-x_{1}-\left(y_{2}\right)_{1}}{\left(\left|x-y_{1}\right|^{2}+\left|y_{1}-y_{2}\right|^{2}\right)^{(2 n+1) / 2}} f\left(y_{1}\right) f\left(y_{2}\right) d y_{1} d y_{2} .
$$

Let

$$
\begin{aligned}
& U_{1}=\left\{x \in \mathbb{R}^{n}:|x| \leq 1, x_{1} \leq x_{i}<0, i=2, \cdots, n\right\}, \\
& V_{1}=\left\{x \in \mathbb{R}^{n}:|x| \leq 1, x_{i} \geq 0, i=1, \cdots, n\right\} .
\end{aligned}
$$

For $0<\varepsilon<1$, let $f_{1}(x)=|x|^{\varepsilon-n} \chi_{V_{1}}(x), f_{2}(x)=|x|^{\frac{\varepsilon-n}{p_{2}}} \chi_{V}(x), w_{1}(x)=$ $|x|^{(\varepsilon-n) p_{1} / p}$ and $w_{2}(x)=1$. Then $v_{\vec{w}}(x)=|x|^{\varepsilon-n}, v_{\vec{w}}^{1-p^{\prime}}=|x|^{(n-\varepsilon)\left(p^{\prime}-1\right)}$ and $w_{1}^{1-p_{1}^{\prime}}=|x|^{(n-\varepsilon) p_{1}^{\prime} / p}$. Similar arguments as the above show that

$$
\left\|\left(R_{1}\right)^{1, *}\right\|_{L^{p^{\prime}}\left(v_{\vec{w}}^{1-p^{\prime}}\right) \times L^{p_{2}}\left(w_{2}\right) \rightarrow L^{p_{1}^{\prime}}\left(w_{1}^{\left.1-p_{1}^{\prime}\right)}\right.} \gtrsim \frac{1}{\varepsilon}=\left[\vec{w}^{1}\right]_{A_{\vec{P} 1}^{p / p_{1}^{\prime}}}^{\prime}=[\vec{w}]_{A_{\vec{P}}} .
$$

Therefore,

$$
\left\|R_{1}\right\|_{L^{p_{1}}\left(w_{1}\right) \times L^{p_{2}}\left(w_{2}\right) \rightarrow L^{p}\left(v_{\vec{w}}\right)}=\left\|\left(R_{1}\right)^{1, *}\right\|_{L^{p^{\prime}}\left(v_{\vec{w}}^{1-p^{\prime}}\right) \times L^{p_{2}}\left(w_{2}\right) \rightarrow L^{p_{1}^{\prime}}\left(w_{1}^{1-p_{1}^{\prime}}\right)} \gtrsim[\vec{w}]_{A_{\vec{P}}} .
$$

This shows the sharpness of the exponent when $p>\max _{i}\left\{p_{i}^{\prime}\right\}$, which completes the proof.

Acknowledgements. The authors thank Carlos Pérez for helping improve the quality of this article.

\section{REFERENCES}

[1] S. Buckley, Estimates for operator norms on weighted spaces and reverse Jensen inequalities, Trans. Amer. Math. Soc., 340 (1993), 253-272.

[2] M. Christ, J.-L. Journé, Polynomial growth estimates for multilinear singular integral operators, Acta Math. 159 (1987) 51-80.

[3] R.R. Coifman and Y. Meyer, On commutators of singular integrals and bilinear singular integrals, Trans. Amer. Math. Soc. 212 (1975) 315-331. 
[4] R.R. Coifman, Y. Meyer, Commutateurs d'intégrales singulières et opérateurs multilinéaires, Ann. Inst. Fourier (Grenoble) 28 (1978) 177-202.

[5] W. Damián, A.K. Lerner and C. Pérez, Sharp weighted bounds for multilinear maximal functions and Calderón-Zygmund operators, http://arxiv.org/abs/1211.5115.

[6] L. Grafakos, Modern Fourier Analysis, Second Edition, Springer-Verlag, 2008.

[7] L. Grafakos, R.H. Torres, Multilinear Calderón-Zygmund theory, Adv. Math. 165 (2002) 124-164.

[8] T. P. Hytönen. The sharp weighted bound for general Calderón-Zygmund operators. Ann. of Math., 175 (2012) 1473-1506.

[9] T. P. Hytönen, The $A_{2}$ theorem: Remarks and complements, preprint, available at http://arxiv.org/abs/1212.3840.

[10] C.E. Kenig, E.M. Stein, Multilinear estimates and fractional integration, Math. Res. Lett. 6 (1999) 1-15.

[11] A. K. Lerner, An elementary approach to several results on the Hardy-Littlewood maximal operator Proc. Amer. Math. Soc., 136 (2008), 2829-2833.

[12] A. K. Lerner, A simple proof of the $A_{2}$ conjecture Int. Math. Res. Not., doi $10.1093 / \mathrm{imrn} / \mathrm{rns} 145$.

[13] A.K. Lerner, S. Ombrosi, C. Pérez, R.H. Torres, R. Trujillo-González, New maximal functions and multiple weights for the multilinear Calderón-Zygmund theory, Adv. Math., 220 (2009), 1222-1264.

[14] H. Lin, Y. Meng, and D. Yang, Weighted estimates for commutators of multilinear Calderón-Zygmund operators with non-doubling measures, Acta Math. Sci., Ser. B, 30 (2010), 1-18.

[15] K. Moen, Sharp weighted bounds without testing or extrapolation, Archiv der Mathematik, 99 (2012) 457-466.

[16] C. Pérez, R.H. Torres, Sharp maximal function estimates for multilinear singular integrals, Contemp. Math., vol. 320 (2003), 323-331.

[17] S. Shi, Z. Fu, and S. Lu, Weighted estimates for commutators of one-sided oscillatory integral operators, Front. Math. China, 6 (2011), 507-516.

[18] Q. Xue and Y. Ding, Weighted estimates for the multilinear commutators of the Littlewood-Paley operators, Sci. China Ser. A, 52 (2009), 1849-1868.

E-mail address: likangwei9@mail.nankai.edu.cn

School of Mathematical Sciences and LPMC, NAnkai University, Tianjin 300071, CHINA

E-mail address: kabe.moen@ua.edu

Department of Mathematics, University of Alabama, Tuscaloosa, AL 35487 , USA

E-mail address: sunwch@nankai.edu.cn

School of Mathematical Sciences and LPMC, NAnkai University, Tianjin 300071, China 